\title{
A Variable-Size Local Domain Approach to Computer Model Validation in Design Optimization
}

\author{
Dorin Drignei and Zissimos Mourelatos \\ Oakland Univ.
}

\author{
Michael Kokkolaras \\ Univ. of Michigan-Ann Arbor \\ Jing Li and Grzegorz Koscik \\ Oakland Univ.
}

\begin{abstract}
A common approach to the validation of simulation models focuses on validation throughout the entire design space. A more recent methodology validates designs as they are generated during a simulation-based optimization process. The latter method relies on validating the simulation model in a sequence of local domains. To improve its computational efficiency, this paper proposes an iterative process, where the size and shape of local domains at the current step are determined from a parametric bootstrap methodology involving maximum likelihood estimators of unknown model parameters from the previous step. Validation is carried out in the local domain at each step. The iterative process continues until the local domain does not change from iteration to iteration during the optimization process ensuring that a converged design optimum has been obtained. The proposed methodology is illustrated using a thermal, one-dimensional, linear heat conduction problem in a solid slab with heat flux boundary conditions.
\end{abstract}

\section{INTRODUCTION}

Design optimization often requires computational analysis or simulation models. These models quantify functional inputoutput relations contained in the objective and constraints. Such models are inexact approximations of the physical world, and so we need to quantify our confidence that designs obtained using simulations will perform as expected when produced. Current practice uses computational models for optimization studies in relatively large design spaces even though the models have been validated only in a small subset of the design space. Within this paradigm, computational models need to be validated in the entire feasible design space in order to obtain high confidence in the results. Computational models are usually validated by calibrating a number of parameters the model is a function of. There is however, inherent uncertainty in both the model calibration parameters and the tests that are conducted to obtain the data to be used in the validation comparisons. For this reason, the model validation procedure can be time consuming and resource intensive.

Due to limited resources, the simulation models are usually validated only at a relatively small number of points in the design space and are then used for optimization studies in the entire design space. This approach can compromise local model accuracy in order to use a single global model which is not calibrated throughout the input space. Li et al. (2010) demonstrated that design optimization using a global model can yield a different and potentially worse optimal design relative to the one obtained by using a model that is calibrated when necessary as the optimization process progresses.

The motivation for the present work is that the aforementioned global model validation may not be necessary. A numerical optimization process creates a sequence of design iterates, whose validity is important only at the optimum. One way to concentrate on the validity of the 
optimal design rather than that of the model is to validate the simulation model at the design iterates as they are generated during the optimization process.

In this paper, we propose a methodology for sequential, calibration-based validation of the simulation model at, and in the vicinity of, the design candidates as they are generated during the optimization process. The goal of such validation is confidence in the resulting design and all intermediate design iterates, rather than in the global performance of the underlying simulation model. A sequential optimization approach is used where the design space at each iteration is much smaller than the entire feasible space. The proposed approach utilizes available testing resources more effectively as it determines the minimum amount of tests required for validation in targeted local domains. It can be also applied to parametric studies to ensure that a design is valid for different operating conditions.

Statistical methods to computer model calibration are available in the literature (Kennedy and O'Hagan, 2001; Bayarri et al., 2007; Higdon et al., 2008; Drignei et al., 2008; Drignei et al., 2010). However, none of these works addresses the number of required test data and a sequential approach to collecting the test data as we propose in this article.

The surrogate management framework by Booker et al. (1999) offers a variation of the main idea presented and implemented here. Booker et al. (1999) generate a sequence of calibrated approximations (metamodels or surrogate models) of the objective function only, which they manage for direct surrogate optimization. They do not assess the validity of their approximations; they simply improve it by calibration during optimization. In the present work, we assess the validity of the design iterates generated by sequential optimization in subsets of the design space by validating the simulation model at these subsets and improving it only if necessary, provided testing can be performed at these points.

\subsection{Background and Previous Research}

Verification and validation (V\&V) of simulation models has been extensively studied during the past decade from researchers, practitioners, professional societies, and government agencies (DoD; Oberkampf and Barone, 2004; Gu and Yang, 2003). A National Science Foundation (NSF) panel on simulation-based engineering science (Oden, 2006), identified $\mathrm{V} \& \mathrm{~V}$ as a core topic. It should be noted that verification is related to computing the simulation model correctly, while validation refers to computing the correct simulation model (Roache, 1998). Verification of a simulation model is an assessment of the accuracy of the solution of simulation model, compared to the mathematical model on which the simulation model is based. Verification is typically done by comparing the solution of the simulation model to the known solution of a particular mathematical model. Verification also provides confidence levels on the accuracy of the simulation models as they are refined, relative to the validity of the underlying mathematical model.

The validation of a simulation model can be viewed as an assessment of accuracy of a simulation model to represent the physical system, in accordance with the user's intentions. This requires an appropriate, well-defined validation metric. Oberkampf, Trucano and Hirsch (2004) provide an excellent review of the state-of-the-art in V\&V methods in computational engineering and physics. They advocate that the validation metric should consider confidence (uncertainty) levels in the computational model, the mathematical model, the tests, and the experimental and model parameters. Validation relies critically on physical test and experimental data and is most effective when validation experiments are constructed carefully to determine the agreement between the simulation model and the experimentally tested physical system, under a well-defined set of conditions.

Model validation approaches can be classified as frequentist (e.g., Oberkampf and Barone, 2004; Esterling and Berger, 2002) or Bayesian (e.g., Kennedy and O'Hagan, 2001; Bayarri et al., 2002). The frequentist approach assumes distributions of model calibration parameters and uses collected data on model predictions to estimate, within confidence intervals, the parameters of the assumed distributions of the model calibration parameters by means of statistical inference. The Bayesian approach assumes an initial prior of the distributions of model calibration parameters and uses observations and predictions to update the previous prior distributions. Typically, the frequentist approach requires much more data to reduce the otherwise unreasonably large confidence intervals. The Bayesian approach does not require large amount of data, and it can be used to incrementally improve distribution approximations as new data become available. For this reason, the Bayesian approach is typically preferred in engineering design.

There are only few methods that focus on model validity for design optimization purposes. The work of Zhang and Mahadevan (2003) initiated a large body of literature (Mahadevan and Rebba, 2005; Rebba and Mahadevan, 2006; Jiang and Mahadevan, 2008a; Jiang and Mahadevan, 2008b; Jiang and Mahadevan, 2009), which uses Bayesian hypothesis testing to accept or reject a design based on model validity. They investigated the effect of model inaccuracies on the reliability requirements of a design using Bayesian formulations to calculate the posterior probability of a design being valid or invalid. Although their approach provides a novel way for design validation, it is limited to design validation with respect to design reliability, and does not 
address the use of validated simulation models in design optimization.

Gunawan and Papalambros (2006) looked into the inaccuracy of a model due to external factors and developed a design confidence metric utilizing Bayesian inference of a binomial process. They then used the metric in reliability-based optimization in order to link confidence, amount of data, and reliability requirement of a potential optimum. There are notable advantages in this approach. First, the availability of a confidence measure enables designers to perform explicit trade-offs between optimality and confidence. Second, the confidence is also directly linked with the amount of data available, and hence the cost associated with it. This can be a valuable tool for the designer conducting a certification effort. Despite these benefits, the method is limited to tackling reliability requirements.

A so-called design-driven approach to validation has been proposed by Chen et al. (2008) using test and model data sampled throughout the design domain. It introduces a statistical prediction model $\hat{Y}$ that associates the test and model data, including a bias term, and it assumes that the design objective $f$ depends on $\hat{Y}$. Because the latter is a random variable, $f$ is also a random variable. For a candidate design $\mathbf{x}^{*}$ to be declared design-valid, it must lead to an improved design objective with high confidence, when compared with the design objective of any other design candidate $\mathbf{x}$. For example, the probabilistic relation

$$
P\left[f\left(\hat{Y}\left(\mathbf{x}^{*}\right)\right)<f\left(\hat{Y}\left(\mathbf{x}_{i}\right)\right)\right]>0.95, \quad \forall \mathbf{x}_{i} \in D, \quad \mathbf{x}_{i} \neq \mathbf{x}^{*}
$$

will indicate that the design candidate $\mathbf{x}^{*}$ is the design-driven optimum with a $95 \%$ confidence. This is an attractive approach. However, it does not provide a metric of statistical agreement between test and model prediction at a particular design candidate. Also, it does not provide a formal measure to ensure that the number of required tests is kept at a minimum.

It should be emphasized that the methodologies of Gunawan and Papalambros (2006) and Chen et al., (2008) are global in the sense that validation is conducted in the entire design domain. In addition, the issues of minimum number of tests at a design candidate and the number of design sites, where designs are conducted, in the global domain are not addressed. We believe that these issues are essential in order to reduce the required resources. Our proposed research addresses both issues.

The paper is organized as follows. Section 2 describes the proposed approach, including the description of the test and prediction models and the definition of the local domains. To illustrate the methodology, Section 3 presents an example involving heat conduction in a solid. Section 4 presents a summary and conclusions.

\section{PROPOSED APPROACH}

This paper proposes a sequential, trust-region-like design optimization methodology, where the simulation model is validated through calibration. Starting with an initial design we conduct optimization within a local domain around that design in which we consider the simulation model to be valid within a given confidence level. Our approach reduces the number of required tests, which are carefully chosen at each design point. In this manner, available resources are utilized effectively while ensuring the validity of the simulation model in the vicinity of the design candidates generated by the optimization process.

The proposed methodology is sequential in that optimization occurs at stages using local, trust-region-like domains; optimization and validation are conducted concurrently in that the model is not validated globally before optimization. The contributions and benefits of the proposed approach include 1) an efficient and effective utilization of available testing resources, 2) an explicit treatment of and accounting for test uncertainty in the validation-driven calibration process, and 3) a quantification of the tradeoff between validation confidence level and local domain size.

Consider a two-dimensional design optimization problem (Figure 1). We start with an initial design $\mathbf{d}=\left(d_{1}, d_{2}\right)$ and determine the minimum number of tests at $\mathbf{d}$ in order to calibrate the simulation model used for analysis so that model predictions match a statistical model of available test data under uncertainty. Minimizing the number of tests is important because tests are usually expensive and time consuming. Subsequently, we determine the size of a local domain around $\mathbf{d}$, so that the simulation model can be considered valid for a certain confidence level. Validation of the simulation model within the local domain means that we are, for example, $95 \%$ confident that there is statistical similarity between the model prediction at each design in the local domain and the test data at the center design d. Each local domain is in general, of rectangular shape with a different range for each design variable.

Ideally, we should compare the model prediction at each design in the local domain with test data at the same design. This is however, impractical because it requires expensive tests at many designs. For this reason, we rely on statistical methods to infer the statistical agreement between model prediction at each design within the local domain and tests at the center design. After the size of the local domain is determined, optimization is performed to obtain the optimal design within the local domain, which completes the first stage of the sequential approach. We then initiate the second stage of the sequential approach using the optimal design of 
the first stage as the initial design of the second stage, and repeat the process by first determining the size of the new local domain (e.g., $D_{2}$ in Figure 1), centered around the new initial design. This sequential optimization process is assumed converged if the Euclidean norm between the optimal designs of two subsequent stages is sufficiently small. In this case, the size of the local domain for two subsequent stages remains the same.

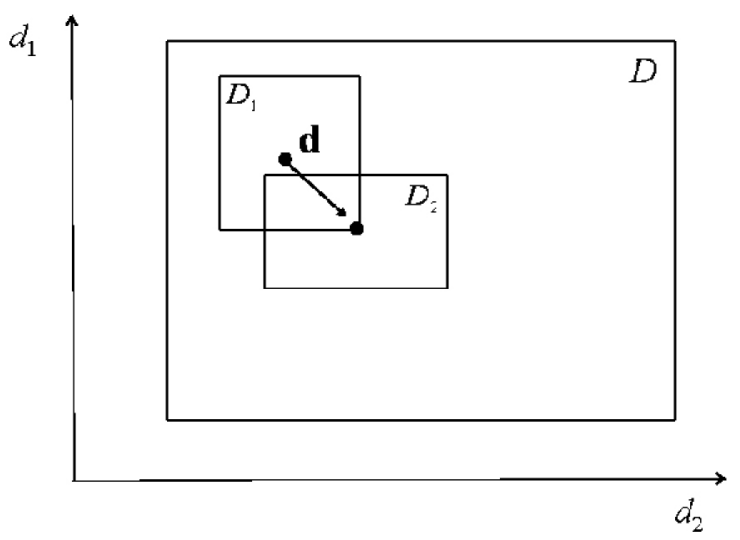

Figure 1. Schematic of proposed sequential optimization approach

The proposed approach ensures validation of the design candidates as they are generated during optimization. The objective of this validation approach is confidence in the resulting design (as well as the intermediate design iterates) rather than in the underlying simulation model. It should be noted that globally validated models may compromise local accuracy. The proposed approach ensures local accuracy as the design optimization process progresses since validationdriven calibration is conducted whenever necessary.

\subsection{Notation and terminology}

Let $y^{r}(\mathbf{d}, \mathbf{p}, \boldsymbol{\tau})$ be the real (superscript $r$ ) and unknown response for design variables $\mathbf{d} \in \mathfrak{R}^{n_{d}}$ and design parameters $\mathbf{p} \in \mathfrak{R}^{n_{p}}$. The argument $\boldsymbol{\tau}$ indicates that the response is, in general, time dependent. Let $y^{t}(\mathbf{d}, \mathbf{p}, \boldsymbol{\tau})$ be the test response (superscript $t$ ), and $y^{m}(\mathbf{d}, \mathbf{p}, \mathbf{c}(\mathbf{d}, \mathbf{p}), \boldsymbol{\tau})$ be the response from the simulation (prediction) model (superscript $m$ ), where $\mathbf{c} \in \mathfrak{R}^{n_{c}}$ is the vector of model calibration parameters. A bold

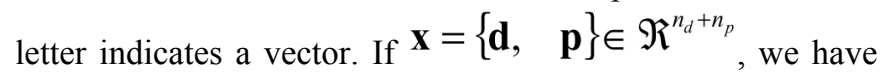
(e.g., Mahadevan and Rebba, 2006):

$$
y^{t}(\mathbf{x}, \tau)=y^{m}(\mathbf{x}, \mathbf{c}, \tau)+\varepsilon(\mathbf{x}, \tau)
$$

where $\varepsilon(\mathbf{x}, \tau)$ is a zero mean random quantity representing the experimental (test) error. Details about $\varepsilon(\mathbf{x}, \boldsymbol{\tau})$ are provided later in the paper. Note that we didn't include a model bias explicitly. However, a bias term (random or not) can be easily be included (e.g. Bayarri et al 2007, Kennedy and O'Hagan 2001), should there be strong evidence for it in specific applications.

At each design point $\mathbf{x}$, multiple tests are usually performed in order to assess the experimental error $\varepsilon(\mathbf{x}, \tau)$. All test responses are included in the vector $\mathbf{y}^{t}(\mathbf{x}, \boldsymbol{\tau})$. The simulation model $y^{m}(\mathbf{x}, \mathbf{c}, \boldsymbol{\tau})$ is also run for different values of $\mathbf{c}$ and all responses are included in the vector $\mathbf{y}^{m}(\mathbf{x}, \mathbf{c}, \boldsymbol{\tau})$.

\subsection{Quantifying test error data}

Because of measurement error and uncontrollable testing conditions, the test responses $\mathbf{y}^{t}(\mathbf{x})$ can differ. Their mean is accounted for in the bias. In order to capture the error variability around the mean statistically, we propose to model $\mathbf{y}^{t}(\mathbf{x})$ as a Gaussian process with mean vector zero and covariance matrix $\Gamma$. The latter may depend on statistical parameters $\boldsymbol{\theta}$ that also need to be estimated. If $\varphi=(\mathbf{c}, \boldsymbol{\theta})$ includes the calibration parameters $\mathbf{c}$ and the statistical parameters $\boldsymbol{\theta}$, the Gaussian probability density function we consider is (Schervish, 1995)

$$
f_{\mathbf{E} / \mathbf{\Phi}}(\boldsymbol{\varepsilon}(\mathbf{x}) \mid \varphi)=\left(\frac{1}{\sqrt{2 \pi}}\right)^{n} \frac{1}{\sqrt{\operatorname{det}(\boldsymbol{\Gamma})}} \exp \left(-\frac{1}{2} \boldsymbol{\varepsilon}(\mathbf{x})^{T} \boldsymbol{\Gamma}^{-1} \boldsymbol{\varepsilon}(\mathbf{x})\right),
$$

where the error $\boldsymbol{\varepsilon}(\mathbf{x})=\mathbf{y}^{t}(\mathbf{x})-\mathbf{y}^{\boldsymbol{m}}(\mathbf{x}, \mathbf{c})$ measures the difference between test and model data for specific values of $\varphi$. The correlation matrix $\boldsymbol{\Gamma}$ models the correlation among the components of the error vector $\boldsymbol{\varepsilon}(\mathbf{x})$, for any design $\mathbf{x}$.

While the Gaussian assumption is widely accepted and works well in many applications, we can check its applicability through a series of plots, such as histograms and quantilequantile (q-q) plots. We can also check the Gaussian assumption through more formal statistical tests, such as Kolmogorov-Smirnov (Rizzo, 2007). If the Gaussian assumption is not appropriate, we can either change the covariance model $\Gamma$ or apply data transformations. The BoxCox data transformations for example can be used in time series analysis when the Gaussian distribution is not appropriate (Brockwell and Davis, 2002).

\subsection{Defining the local domains}

We propose to use the set of available test data $\mathbf{y}^{t}(\mathbf{x})$, obtained during the process of validating the simulation model at design $\mathbf{x}$, to determine the size of the local domain around $\mathbf{x}$, within which the simulation model can be assumed valid with a given confidence level. Design optimization will then be conducted within this local domain so that the next design candidate can be found. 
When the design $\mathbf{x}$ is fixed, the likelihood function of the parameter vector $\varphi$ given the available test data, is

$$
L(\varphi \mid \mathbf{\varepsilon}(\mathbf{x})):=f_{\mathbf{E} / \mathbf{\Phi}}(\boldsymbol{\varepsilon}(\mathbf{x}) \mid \varphi)
$$

To define the size of the domain around $\mathbf{x}$, within which the model is considered valid for a given confidence level, we propose to use the extended likelihood $L(\mathbf{x}, \varphi \mid \varepsilon(\mathbf{x}))$, which has the same form as $L$ in Equation (3), but with $\mathbf{x}$ as an additional statistical parameter. We then use a parametric bootstrap approach (Efron and Tibshirani, 1993; Rizzo, 2007) based on simulated data.

Specifically, we generate sample data $\overline{\mathbf{\varepsilon}}(\mathbf{x})$ (e.g., 200 different time history realizations of $\varepsilon(\mathbf{x})$ ) from the Gaussian distribution of $\varepsilon(\mathbf{x})$ which has a zero mean vector and a covariance matrix $\Gamma$. Thus, we obtain simulated test data $\bar{y}^{t}(\mathbf{x})$ as

$$
\bar{y}^{t}(\mathbf{x})=y^{m}(\mathbf{x}, \hat{\mathbf{c}})+\bar{\varepsilon}(\mathbf{x})
$$

The bar in the above equation indicates simulated (not actual) data. We then re-estimate the statistical model (i.e. recalibrate the simulation model) by maximizing the extended likelihood, using the simulated data $\bar{y}^{t}(\mathbf{x})$ instead of actual test data $y^{t}(\mathbf{x})$. This will yield the most likely values of $\bar{\psi}=(\overline{\mathbf{x}}, \bar{\varphi})$. We repeat this process many times, say $B=500$, to obtain the bootstrap finite sample $\left\{\bar{x}_{i, b}\right\}_{b=1: B}$ of statistical copies of each component $x_{i}$ of $\mathbf{x}$. Then, the simulation-based $90 \%$ confidence interval for example, for $x_{i}$ is $\left(\bar{x}_{i, L}, \bar{x}_{i, U}\right)$ where $\bar{x}_{i, L}$ and $\bar{x}_{i, U}$ are the $5^{\text {th }}$ and $95^{\text {th }}$ percentiles of the finite sample $\left\{\bar{x}_{i, b}\right\}_{b=1: B}$. These bootstrap percentiles can be improved by bias-correcting them, a process that results in a bootstrap sample centered approximately at $\mathbf{x}$ (e.g. Efron and Tibshirani, 1993).

The above process defines a local domain of hyperrectangular shape. The length of the $i^{\text {th }}$ side of the hyperrectangle is equal to $\left(\bar{x}_{i, U}-\bar{x}_{i, L}\right)$. It should be noted that the length $\left(\bar{x}_{i, U}-\bar{x}_{i, L}\right)$ depends on the local geometrical features of the computer model $y^{m}(\mathbf{x}, \mathbf{c}, \boldsymbol{\tau})$ with respect to $\mathbf{x}$ as indicated by the inverse of the Hessian matrix $H(\mathbf{x})=\frac{\partial^{2} y^{m}(\mathbf{x}, \mathbf{c}, \tau)}{\partial x_{i} \partial x_{j}}$

at design $\mathbf{x}$. Statistical theory (Schervish, 1995) shows that the maximum likelihood estimator $\hat{\mathbf{x}}$ of $\mathbf{x}$ has a distribution that is approximately Gaussian with a covariance matrix of $[-H(\mathbf{x})]^{-1}$. Therefore, the approximate standard errors $S . E .\left(\hat{x}_{i}\right)$ of the components $\hat{x}_{i}$ of $\hat{\mathbf{x}}$ are equal to the square root of the elements on the main diagonal of $[-H(\mathbf{x})]^{-1}$. Statistical theory also indicates that the confidence interval of component $\hat{x}_{i}$ (i.e. $\hat{x}_{i} \pm 1.96 S . E .\left(\hat{x}_{i}\right)$ is approximately equal to the parametric bootstrap confidence interval $\left(\bar{x}_{i, U}-\bar{x}_{i, L}\right)$ described above (Efron and Tibshirani, 1993).

The interval $\left(\bar{x}_{i, L}, \bar{x}_{i, U}\right)$ specifies the length of the $i^{\text {th }}$ side of the local domain within which the computer model is considered valid; i.e., we are confident about the statistical similarity between the available tests and the model predictions. The design optimization problem is solved in this local domain. Note that we can simultaneously define concentric local domains of different size by considering different percentiles (e.g. $2.5^{\text {th }}$ and $97.5^{\text {th }}$ percentiles for $95 \%$ confidence level). Therefore, our proposed method provides confidence regions of different levels (e.g. 95\%, 90\%, 80\%, etc). The user can choose a variable confidence level, and therefore a variable local domain size throughout the optimization procedure, depending on his/her particular application and available resources. This is analogous to choosing the step size in a nonlinear optimization; some regions can support a larger step size, improving the efficiency of the optimization algorithm.

The following algorithm summarizes the method developed in this paper.

1. Choose an initial design $\mathbf{x}^{(0)}$, where both test data and model prediction data are generated.

2. The computer model is calibrated at the current design.

3. Obtain the local domain surrounding the previous design.

4. Solve the constrained optimization in the local domain from Step 3.

\section{Repeat steps 2 through 4 until convergence is achieved.}

\section{A THERMAL PROBLEM EXAMPLE}

This example considers heat conduction in a solid (Dowding et al., 2008). It involves a safety device which is a material layer of thickness $L$ exposed to a heat flux $q$. The temperature response of the device is modeled using one-dimensional heat conduction through a slab (Figure 2). A specified heat flux on the $s=0$ face and an adiabatic condition $(q=0)$ on the $s=L$ face are used as boundary conditions. The thermal conductivity $k$ in $W / m^{\circ} C$, the volumetric heat capacity $\rho C_{p}$ in $\mathrm{J} /{ }^{\circ} \mathrm{C} \mathrm{m}$, and the initial condition $T(s, 0)=T_{i}=25$ in ${ }^{\circ} \mathrm{C}$ are 


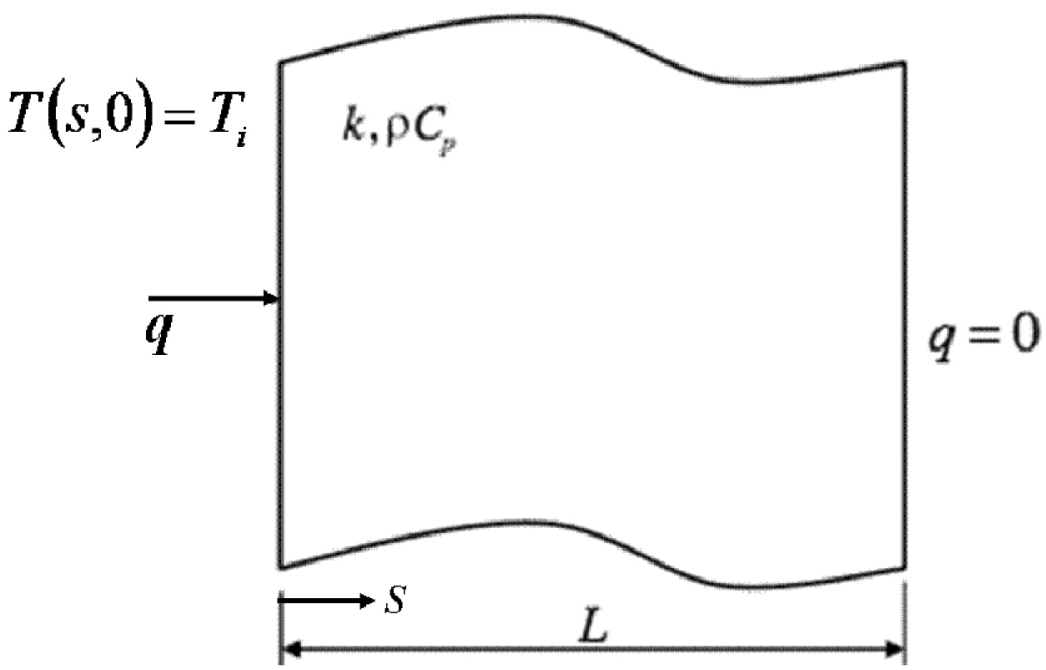

Figure 2. Schematic of heat conduction problem

assumed constant. The initial temperature $T_{i}$ remains fixed throughout the example.

The analytical solution for the temperature $T(q, L, s, t)$ over space $s$ and time $t>0$ is expressed as

$T(q, L, s, t)=T_{i}+\frac{q L}{k}\left[\frac{t k / \rho C_{p}}{L^{2}}+\frac{1}{3}-\frac{s}{L}+\frac{1}{2}\left(\frac{s}{L}\right)^{2}-\right.$

$$
\left.\frac{2}{\pi^{2}} \sum_{n} \frac{1}{n^{2}} \exp \left(-n^{2} \pi^{2} \frac{k / \rho C_{p}}{L^{2}}\right) \cos \left(n \pi \frac{s}{L}\right)\right]
$$

The following optimization problem is solved:

$$
\max _{\mathbf{x}=(q, L)} \frac{q / 4000}{L / 0.03}
$$

such that:

$$
\begin{gathered}
\min _{\tau} T\left(q_{1}, L_{1}, \tau\right) \geq 25.01 \\
500 \leq q \leq 4000 \\
0.01 \leq L \leq 0.03 \\
t \geq 100 \\
q_{1}=4000-q, \quad L_{1}=0.03-L
\end{gathered}
$$

where the temperature $T(q, L, \tau)$ with $\tau=(s, t)$, appears only in the nonlinear constraint. The objective is to maximize the scaled ratio $q / L$.

The following steps outline our proposed variable-size local domain approach to computer model validation for this onedimensional thermal problem:
Step 1: Choose an initial design $\mathbf{x}^{(0)}=\left(q^{(0)}, L^{(0)}\right)$, where both test data and model prediction data are generated.

The initial design $\mathbf{x}^{(0)}=(2000,0.02)$ is chosen (see Figure 4). The model prediction data $y^{m}(\mathbf{x}, \mathbf{c}, \tau)$ for the temperature $T$ are generated using Equation (5). For this example, hypothetical test data $y^{t}(\mathbf{x}, \tau)$ are generated using Equation (1) as

$$
y^{t}(\mathbf{x}, \tau)=y^{m}(\mathbf{x}, \mathbf{c}, \tau)+\varepsilon(\mathbf{x}, \tau)
$$

based on the likelihood of Equation (3) with $\sigma^{2}=1$ and $\boldsymbol{\Gamma}=$ $\mathbf{C}_{T} \otimes \mathbf{C}_{s}$. Figure 3 shows the model prediction data (blue) and the hypothetical test data (green). The latter resemble and retain the smoothness properties of the prediction model data. To generate such smooth test data, we discretized the time interval $[0,1000](\mathrm{sec})$ and the space interval $[0, L](\mathrm{cm})$ using $\left(t_{i}\right)_{i=1}^{20}$ and $\left(s_{i}\right)_{i=1}^{20}$ equally-spaced time and space points, respectively. A Kronecker product of temporal and spatial correlation matrices given by

$\mathbf{C}_{T}=\left[\exp \left(-\theta_{t}\left(t_{i}-t_{j}\right)^{2}\right)\right]_{i, j=1}^{20}$ and $\mathbf{C}_{S}=\left[\exp \left(-\theta_{s}\left(s_{i}-s_{j}\right)^{2}\right)\right]_{i, j=1}^{20}$,

with $\theta_{t}=\theta_{s}=10$ is then used to provide a correlation structure.

These correlations are distance-based, in which data are less correlated at space (or time) points farther apart. The Kronecker product is a common statistical strategy called separability to join together correlations in several dimensions (e.g. space and time). In this example, we use one test per design point. However, several tests per design point can be also used. 


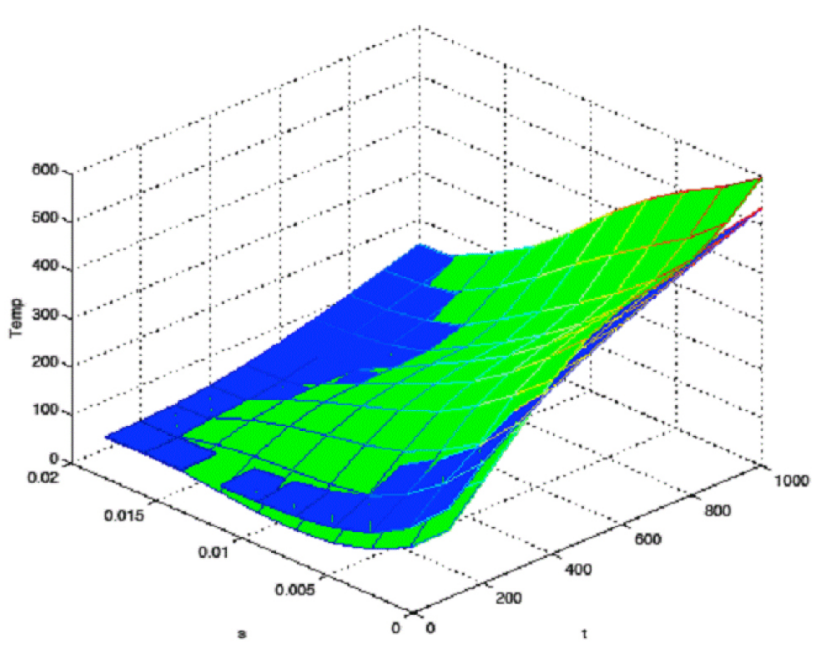

Figure 3. Hypothetical test data (green) and model prediction data (blue)

Step 2: The computer model is calibrated at the current design.

In this step we determine the most likely values of the computer model parameters $\varphi=\left(k, \rho C_{p}, \theta_{t}, \theta_{s}\right)$ so that the test and model prediction data are statistically similar. This ensures that the computer model is validated at the current design. A statistical model of the hypothetical test data is built using the likelihood of Equation (3) and its statistical parameters $\varphi$ are estimated by maximum likelihood as $\hat{\varphi}=\left(\hat{k}, \rho \hat{C}_{p}, \hat{\theta}_{t}, \hat{\theta}_{s}\right)=(0.018,280340,12.425,13.511)$.

\section{Step 3: Obtain the local domain.}

Subsequently, $B=500$ test data are generated from Equation (4) and a statistical model with an extended likelihood (i.e. including $\mathbf{x}$ as an additional statistical parameter) is reestimated for each of the $B=500$ test data. Thus, we obtain a bootstrap sample for each statistical parameter. Among these parameters, $\mathbf{x}$ is the parameter of interest. We use $B=500$ bias-corrected bootstrap replicates of $\mathbf{x}$ in order to obtain its $5^{\text {th }}$ and $95^{\text {th }}$ percentiles. These percentiles provide the size of the local domain surrounding the starting point $\mathbf{x}^{(0)}=$ $(2000,0.02)$ in Figure 4.

Step 4: Solve the constrained optimization problem of Equation (6) in the local domain from Step 3.

The optimization problem of Equation (6) is solved and the new optimum design $\mathbf{x}^{(1)}=\left(q^{(1)}, L^{(1)}\right) \approx(2242,0.017)$ is calculated. This optimum is located at the lower right corner of the local domain (Figure 4).
Step 5: Repeat steps 2 through 4 until convergence is achieved.

After several iterations, the final optimum design $\mathbf{x}=(q, L)=$ $(3611,0.0121)$ is obtained. Figure 4 shows the optimization path along with the local domains and their respective sizes. The contours in Figure 4 are linear, corresponding to the objective function $\frac{q / 4000}{L / 0.03}=K$ for different values of $K$.

According to Section 2.3, the local domains are rectangular. The length of each side depends on the local geometrical features of the computer model $y^{m}(\mathbf{x}, \mathbf{c}, \tau)$ with respect to $\mathbf{x}$ as indicated by the inverse of the Hessian matrix at design $\mathbf{x}$ (Schervish, 1995), not on the linearity of the objective

$$
\text { function } \frac{q / 4000}{L / 0.03} \text { with respect to } \mathbf{x}=(q, L) \text {. }
$$

It should be noted that the temperature constraint (black dotted line in Figure 4) changes from iteration to iteration, because it depends on the calibration parameters whose estimated values are different in each local domain.

\section{SUMMARY AND CONCLUSIONS}

We presented a methodology for validating computer models as they are used during the simulation-based optimization process. The presented methodology requires the validity of the prediction models only at designs generated during a sequential optimization approach where sub-optimization problems are solved within local design domains that are subsets of the entire domain. This is different from the current practice where a-priori validation of a simulation model is performed throughout the entire design space and then used to obtain the optimum design. The proposed methodology to computer model validation using a variablesize local domain approach was demonstrated using an onedimensional, linear heat conduction in a solid slab thermal problem with heat flux boundary conditions. The methodology can determine the minimum number of tests at a design candidate and the number of design sites, where designs are conducted. We believe that this is essential in order to reduce the resources required to obtain the optimum design using a validated computer model. 


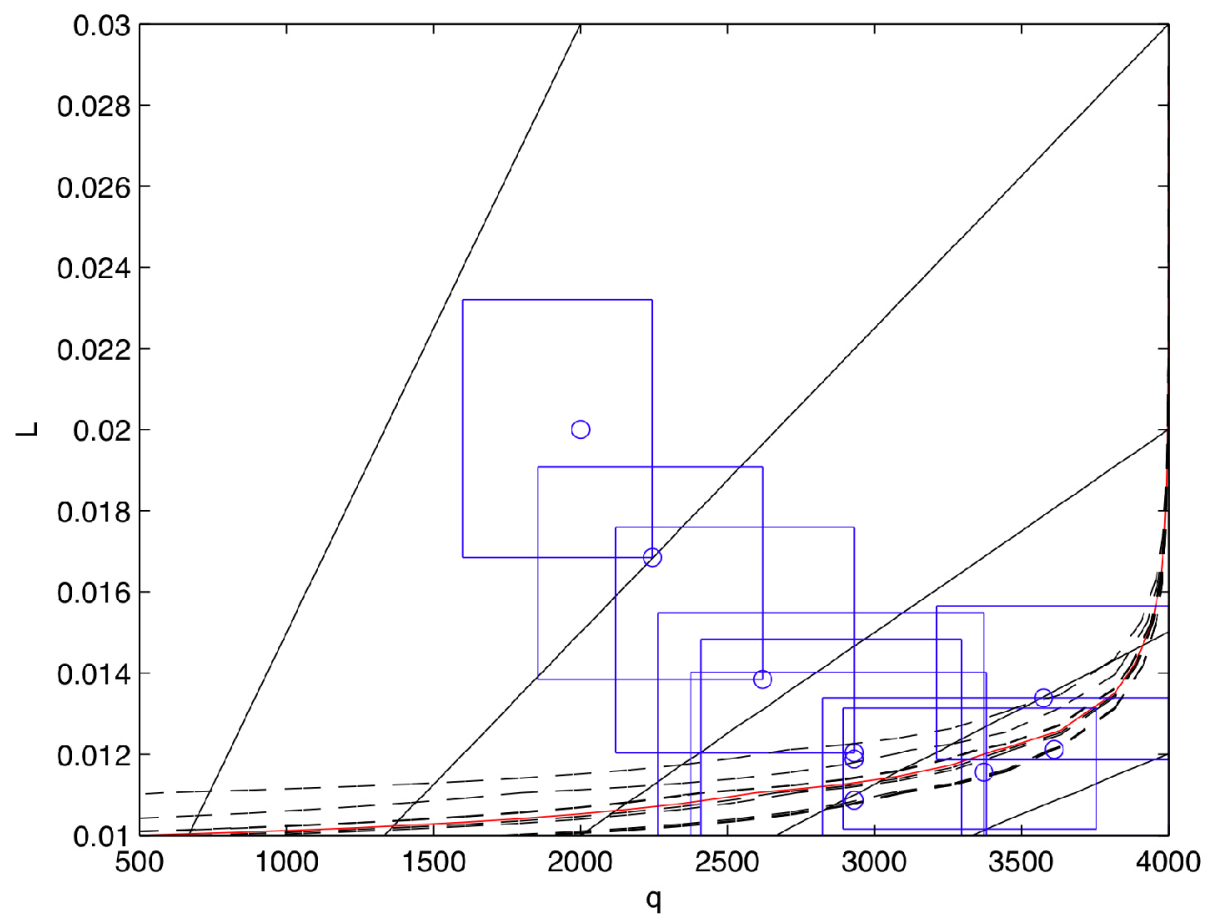

Figure 4. Optimization path of heat conduction problem

\section{REFERENCES}

Bayarri, M. J., Berger, J. O., Higdon, D., Kennedy, M. C., Kottas, A., Paulo, R., Sacks, J., Cafeo, J. A., Cavendish, J., Lin, C. H. and Tu, J., "A Framework for Validation of Computer Models," Foundations for Verification and Validation in $21^{\text {st }}$ Century Workshop, Johns Hopkins University, October, 2002.

Bayarri, M. J., Walsh, D., Berger, J. O., Cafeo, J., GarciaDonato, G., Liu, F., Palomo, J., Parthasarathy, R. J., Paulo, R. and Sacks, J., "Computer Model Validation with Functional Output," Annals of Statistics, 35, 1874-1906, 2007.

Booker, A. J., Dennis, J. E.Jr., Frank, P. D., Serafini, D. B., Torczon, V. and Trosset, M. W., "A Rigorous Framework by Surrogates for Optimization of Expensive Functions," Structural Optimization, 17, 1-13, 1999.

Brockwell, P. J. and Davis, R. A., Introduction to Time Series and Forecasting, Second Edition, Springer, 2002.

Chen, W., Xiong, Y., Tsui, K.-L. and Wang, S., “A DesignDriven Validation Approach Using Bayesian Prediction Models," Journal of Mechanical Design, 130, 021101 (12 pages), 2008.

DoD Directive No. 5000.61, "Modeling and Simulation (M\&S), Verification, validation, and Accreditation (VV\&A)," Defense Modeling and Simulation Office, www.dmso.mil/docslib.
Dowding, K.J., Pilch, M. and Hills, R.G. (2008), Formulation of the thermal problem, Comput. Methods Appl. Mech. Engrg. 197, 2385-2389

Drignei, D., Forest, C. and Nychka, D., "Parameter Estimation for Computationally Intensive Nonlinear Regression with an Application to Climate Modeling," Annals of Applied Statistics, 2, 1217-1230, 2008.

Drignei, D., Mourelatos, Z. P. and Rebba, R., "Parameter Screening in Dynamic Computer Model Calibration Using Global Sensitivities," Proceedings of the ASME 2010 IDETC/ CIE, August 15-18, Montreal, Quebec, Canada, DETC2010-28343, 2010.

Efron, B. and Tibshirani, R. J., An introduction to the Bootstrap, Chapman \& Hall, New York, 1993.

Easterling, R. G. and Berger, J. O., "Statistical Foundations for the Validation of Computer Models," Presentation at Computer Model Verification and Validation in the $21^{\text {st }}$ Century Workshop, Johns Hopkins University, 2002.

Gu, L. and Yang, R. J. "Recent Applications on ReliabilityBased Optimization of Automotive Structures," SAE Technical Paper 2003-01-0152, 2003, doi: 10.4271/2003-01-0152.

Gunawan, S. and Papalambros, P. Y., "A Bayesian Approach to Reliability-Based Optimization with Incomplete Information," Journal of Mechanical Design, 128(4), 909-918, 2006. 
Higdon, D., Gattiker, J., Williams, B. and Rightley, M., "Computer Model Calibration using High Dimensional Outputs,” J. Am. Statist. Assoc., 103, 570-583, 2008.

Jiang, X., and Mahadevan, S., "Bayesian Validation Assessment of Multivariate Computational Models," Journal of Applied Statistics, 35(1), 49-65, 2008a.

Jiang, X., and Mahadevan, S., "Bayesian Wavelet Method for Multivariate Model Assessment of Dynamic Systems," Journal of Sound and Vibration, 312(4-5), 694-712, 2008b.

Jiang, X., and Mahadevan, S., "Bayesian Structural Equation Modeling for Hierarchical Model Validation," Reliability Engineering and System Safety, 94(4), 796-809, 2009.

Kennedy, M. C. and O'Hagan, A., "Bayesian Calibration of Computer Models,” J. R. Statist. Soc. B, 63, 425-450, 2001.

Li, J., Mourelatos, Z. P., Kokkolaras, M., Papalambros, P. and Gorsich, D., "Validating Designs Through Sequential Simulation-Based Optimization," Proceedings of the ASME 2010 IDETC/CIE, August 15-18, Montreal, Quebec, Canada, DETC2010-28431, 2010.

Mahadevan, S. and Rebba, R., "Validation of Reliability Computational Models using Bayes Networks," Reliability

Engineering and System Safety, 87, 223-232, 2005.

Mahadevan, S. and Rebba, R., "Inclusion of Model Errors in Reliability-Based Optimization," Journal of Mechanical Design, 128(4), 936-944, 2006.

Oberkampf, W. and Barone, M., "Measures of Agreement Between Computation and Experiment: Validation Metrics," $34^{\text {th }}$ AIAA Fluid Dynamics Conference and Exhibit, Portland, OR, June 28-July 1, AIAA-2004-2626, 2004.

Oberkampf, W. L, Trucano, T. G. and Hirsch, C., "Verification, Validation, and Predictive Capability in Computational Engineering and Physics," Applied Mechanics Review, 57(5), 345-384, 2004.

Oden, J. T.chair, "Revolutionizing Engineering Science Through Simulation: The NSF Blue Ribbon Panel on Simulation-Based Engineering Science," National Science Foundation, 2006.

Rizzo, M. L., Statistical Computing with R, Chapman and Hall, 2007.
Roache, P. J., Verification and Validation in Computational Science and Engineering, Hermosa Publishing, Albuquerque, NM, 1998.

Schervish, M. J., Theory of Statistics, Springer, 1995.

Zhang, R. and Mahadevan, S., "Bayesian Methodology for Reliability Model Acceptance," Reliability Engineering \& System Safety, 80, 95-103, 2003.

\section{CONTACT INFORMATION}

\section{Dorin Drignei}

Department of Mathematics and Statistics

Oakland University

Rochester, MI 48309

drignei@oakland.edu

Zissimos P. Mourelatos

Mechanical Engineering Department

Oakland University

Rochester, MI 48309

mourelat@oakland.edu

\section{Grzegorz Koscik}

Department of Mathematics and Statistics

Oakland University

Rochester, MI 48309

gkoscik@oakland.edu 